\title{
A Linear Estimator for Network Localization Using Integrated RSS and AOA Measurements
}

\author{
Slavisa Tomic ${ }^{(1)}$, Marko Beko, and Milan Tuba
}

\begin{abstract}
This letter addresses the problem of simultaneous localization of multiple targets in three-dimensional cooperative wireless sensor networks. To this end, integrated received signal strength and angle of arrival measurements are employed. By exploiting the convenient nature of spherical representation of the considered problem, the measurement models are linearized and a sub-optimal estimator is formulated. Unlike the maximum likelihood estimator, which is highly non-convex and difficult to tackle directly, the derived estimator is quadratic and has a closed-form solution. Its computational complexity is linear in the number of connections and its accuracy surpasses the accuracy of existing ones in all considered scenarios.
\end{abstract}

Index Terms-Network localization, received signal strength (RSS), angle of arrival (AOA), weighted least squares (WLS).

\section{INTRODUCTION}

A CCURATE localization of network devices attracts plenty of attention in the research society, since it is important in many applications [1]-[9]. Being able to perform this task using already deployed terrestrial technologies is one of the main requirements. Thus, existing schemes typically take advantage of measurements acquired from received signal strength (RSS), angle of arrival (AOA), etc., or their combination [1], [3]-[8].

Notable advance has been made in developing RSS-AOA localization algorithms recently [10]-[19]. Nevertheless, most of these methods are designed for non-cooperative localization only [10]-[14], where a single target is able to communicate with all available anchors is located at a time. In [15], [16], the authors presented two second-order cone programming estimators for cooperative localization. However, these estimators were designed for distributed (iterative) implementation that suffers from error propagation. Moreover, their iterative nature

Manuscript received October 26, 2018; revised December 16, 2018; accepted January 7, 2019. Date of publication January 10, 2019; date of current version January 24, 2019. This work was supported by Fundação para a Ciência e a Tecnologia under Projects UID/MULTI/04111/0213, UID/MULTI/04111/0216, UID/EEA/00066/2013, and foRESTER PCIF/SSI/0102/2017, and in part by Program Investigador FCT under Grant IF/00325/2015. The work of M. Tuba was supported by the Ministry of Education, Science and Technological Development of Republic of Serbia under Grant No. III-44006. The associate editor coordinating the review of this manuscript and approving it for publication was Dr. Ioannis D. Schizas. (Corresponding author: Slavisa Tomic.)

$\mathrm{S}$. Tomic is with the COPELABS, Universidade Lusófona de Humanidades e Tecnologias, Lisboa 1749-024, Portugal, and also with the ISR/IST, LARSyS, Universidade de Lisboa, Lisbon 1049-001, Portugal (e-mail: slavisa. tomic@ulusofona.pt).

M. Beko is with the COPELABS, Universidade Lusófona de Humanidades e Tecnologias, Lisboa 1749-024, Portugal, and also with the CTS/UNINOVA, Caparica 2829-516, Portugal (e-mail: beko.marko@ulusofona.pt).

M. Tuba is with the Department of Technical Sciences, State University of Novi Pazar, Novi Pazar 36300, Serbia (e-mail: tuba@np.ac.rs).

Digital Object Identifier 10.1109/LSP.2019.2892225 does not make them very suitable for real-time applications. Two semidefinite programming (SDP) estimators for cooperative, centralized localization were presented in [17], [18]. These SDPs have very high computational cost that increases significantly with the network size. A linear least squares (LLS) method based on a simple geometrical approach, where anchors are used as reference points and RSS/AOA measurements are employed to estimate the distance/direction towards the target of interest was proposed in [19]. Information from two-hop neighbors was exploited to localize targets without a direct link to anchors.

The RSS-AOA localization algorithms for cooperative networks presented in [15]-[18] are based on sophisticated mathematical tools which makes them computationally burdensome. On the other hand, LLS algorithm in [19] has linear computational complexity, but fails to work if a target has no anchor neighbors within two hops. In sharp contrast to these algorithms, we propose here a novel one, with linear computational complexity in the number of edges, that does not require any additional conditions (such as in [19]) to work. By exploiting the available AOA observations, the proposed algorithm benefits from a natural formulation using spherical coordinates. Not only that it is computationally light, but our numerical results show that it outperforms the existing ones.

\section{PROBLEM Formulation}

Let us consider a connected 3-D network with $M$ targets (sensors we want to localize) and $N$ anchors (sensors whose locations are known a priori, used as reference points) whose true locations are denoted by $\boldsymbol{x}_{i} \in \mathbb{R}^{3}$ for $i=1, \ldots, M$ and $\boldsymbol{a}_{j} \in \mathbb{R}^{3}$ for $j=1, \ldots, N$, respectively. Owing to the sensors' battery lives, it is assumed that all sensors have limited communication range, $R$. Hence, some pairwise distance $\left(d_{i j}\right)$ and direction (both azimuth, $\phi_{i j}$, and elevation, $\alpha_{i j}$, angles) measurements among sensors are obtained through RSS and AOA estimation techniques, as illustrated in Fig. 1. Our goal is to simultaneously determine the unknown locations of all targets by resorting to these observations and the reference locations of the anchors. In an idealistic noise-free and non-degenerate scenario, a single target can be located unambiguously with: (1) four RSS observations to four different anchors, (2) two AOA observations (both azimuth and elevation) to two different anchors and (3) a single hybrid RSS-AOA observation to an anchor. However, due to limited communication range of the sensors, only some targets can directly communicate with anchors and target cooperation is required, i.e., besides anchors, targets are also allowed to communicate with targets within their communication range. 


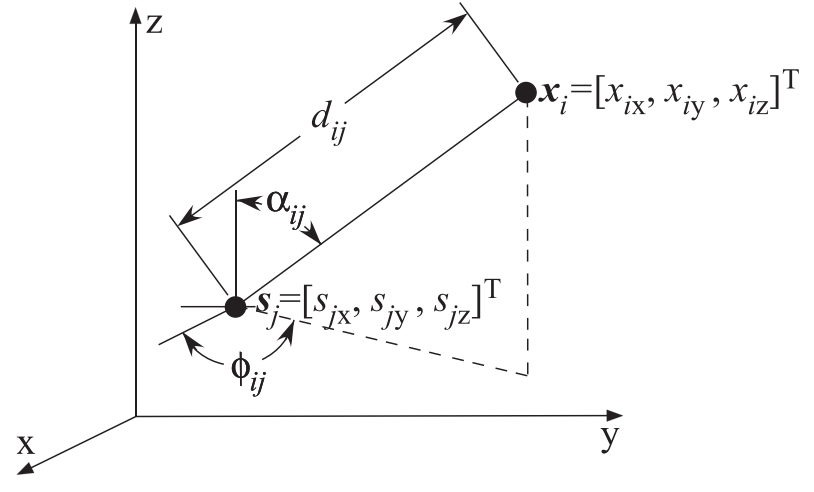

Fig. 1. Model illustration in a 3-D space.

The RSS measurement model can be replaced with the path loss model by using the relationship $L_{i j}=10 \log _{10} \frac{P_{T}}{P_{i j}}(\mathrm{~dB})$, where $L_{i j}$ and $P_{i j}$ are respectively the path loss and received power between two sensors $i$ and $j$, and $P_{T}$ is the transmission power of a sensor, [1], [13], as:

$$
L_{i j}=L_{0}+10 \gamma \log _{10} \frac{d_{i j}}{d_{0}}+n_{i j}, \text { for }(i, j) \in \mathcal{E},
$$

where $d_{i j}=\left\|\boldsymbol{x}_{i}-\boldsymbol{s}_{j}\right\|$ with $\boldsymbol{s}_{j}$ denoting the $j$-th neighboring sensor (either an anchor or a target), $L_{0}$ denotes the path loss value at a short reference distance $d_{0}\left(d_{i j} \geq d_{0}\right), \gamma$ is the path loss exponent (PLE) that indicates the rate at which the path loss increases with distance, and $n_{i j}$ is the log-normal shadowing terms modeled as a zero-mean Gaussian random variable with variance $\sigma_{n_{i j}}^{2}$, i.e., $n_{i j} \sim \mathcal{N}\left(0, \sigma_{n_{i j}}^{2}\right)$. Furthermore, the tuple set $\mathcal{E}=\left\{(i, j):\left\|\boldsymbol{x}_{i}-\boldsymbol{s}_{j}\right\| \leq R\right.$, for $i=1, \ldots, M, j=$ $1, \ldots, M, M+1, \ldots, M+N, i \neq j\}$ denotes the existence of an edge between two sensors.

The azimuth and the elevation angles can be respectively modeled as [10], [18]:

$$
\begin{aligned}
& \phi_{i j}=\tilde{\phi}_{i j}+m_{i j}, \text { for }(i, j) \in \mathcal{E}, \\
& \alpha_{i j}=\tilde{\alpha}_{i j}+v_{i j}, \text { for }(i, j) \in \mathcal{E},
\end{aligned}
$$

where $\tilde{\phi}_{i j}=\tan ^{-1}\left(\frac{x_{i \mathrm{y}}-s_{j \mathrm{y}}}{x_{i \mathrm{x}}-s_{j \mathrm{x}}}\right), \tilde{\alpha}_{i j}=\cos ^{-1}\left(\frac{x_{i \mathrm{z}}-s_{j \mathrm{z}}}{\left\|\boldsymbol{x}_{i}-\boldsymbol{s}_{j}\right\|}\right), m_{i j}$ and $v_{i j}$ are the measurement errors of the azimuth and the elevation angles respectively, modeled as a zero-mean von Mises random variables with the concentration parameters $\kappa_{m_{i j}}, \kappa_{v_{i j}} \in$ $[0, \infty)$, i.e., $m_{i j} \sim V M\left(0, \kappa_{m_{i j}}\right)$ and $v_{i j} \sim V M\left(0, \kappa_{v_{i j}}\right)$. Note that this is in sharp contrast to the existing approaches for joint RSS and AOA localization (both non-cooperative [10]-[14] and cooperative [17]-[19]), where the measurement errors of angle observations were considered as Gaussian random variables. The von Mises distribution is a circular analogue of the Gaussian one, and since we are dealing here with directional data, it comes more natural to consider this distribution rather than the Gaussian one [20], [21]. Furthermore, the Gaussian distribution is not generally suitable for AOA estimates, since it has an infinite support instead of a periodic one $(2 \pi)$ over the angular domain [20]. Without loss of generality, we assume that $\kappa_{m_{i j}}=\kappa_{v_{i j}}=\kappa_{i j}$. It is also worth mentioning that the mean direction and the concentration parameter of the von Mises distribution can be related to the mean and variance in the Gaussian distribution [20], [21].

For the sake of simplicity, we stack all unknown vectors into a single matrix, i.e., $\boldsymbol{X}=\left[\boldsymbol{x}_{1}, \ldots, \boldsymbol{x}_{M}\right],\left(\boldsymbol{X} \in \mathbb{R}^{3 \times M}\right)$. From (1), the conditional probability density function (PDF) of an RSS observation is given by

$$
\begin{aligned}
f_{L_{i j}}\left(L_{i j} \mid \boldsymbol{X}\right)= & \frac{1}{\sqrt{2 \pi \sigma_{n_{i j}}^{2}}} \\
& \times \exp \left\{-\frac{\left(L_{i j}-L_{0}-\log _{10} \frac{d_{i j}}{d_{0}}\right)^{2}}{2 \sigma_{n_{i j}}^{2}}\right\} .
\end{aligned}
$$

Similarly, from (2) we can write the conditional PDF of an azimuth and elevation observations as

$$
\begin{aligned}
& f_{\phi_{i j}}\left(\phi_{i j} \mid \boldsymbol{X}\right)=\frac{1}{2 \pi I_{0}\left(\kappa_{i j}\right)} \exp \left\{\kappa_{i j} \cos \left(\phi_{i j}-\tilde{\phi}_{i j}\right)\right\}, \\
& f_{\alpha_{i j}}\left(\alpha_{i j} \mid \boldsymbol{X}\right)=\frac{1}{2 \pi I_{0}\left(\kappa_{i j}\right)} \exp \left\{\kappa_{i j} \cos \left(\alpha_{i j}-\tilde{\alpha}_{i j}\right)\right\},
\end{aligned}
$$

where $I_{k}(\bullet)$ is the modified Bessel function of first kind of order $k$ [20], [21].

By maximizing (3) and (4), a maximum likelihood (ML) estimator of $\boldsymbol{X}$ is obtained. Although the ML solution is asymptotically optimal, it is highly non-convex with no closed-form solution. Therefore, we propose a sub-optimal approach in Section III, whose solution is given in closed-form.

\section{The Proposed WLS Estimator}

What has gone unnoticed in [18], where a heuristic approach was taken, is that from (3), the distance that best estimates $\left\|\boldsymbol{x}_{i}-\boldsymbol{s}_{j}\right\|$ in the ML sense is

$$
\widehat{d}_{i j}=d_{0} 10^{\frac{L_{i j}-L_{0}}{10 \gamma}} .
$$

Similarly, from (4), the angles which best estimate $\phi_{i j}$ and $\alpha_{i j}$ in the ML sense are respectively

$$
\begin{aligned}
& \widehat{\phi}_{i j}=\tan ^{-1}\left(\frac{x_{i \mathrm{y}}-s_{j \mathrm{y}}}{x_{i \mathrm{x}}-s_{j \mathrm{x}}}\right), \\
& \widehat{\alpha}_{i j}=\cos ^{-1}\left(\frac{x_{i \mathrm{z}}-s_{j \mathrm{z}}}{\left\|\boldsymbol{x}_{i}-\boldsymbol{s}_{j}\right\|}\right),
\end{aligned}
$$

since (4) is maximized when the cosines in the arguments are equal to 1 , i.e., when $\widehat{\phi}_{i j}=\tilde{\phi}_{i j}$ and $\widehat{\alpha}_{i j}=\tilde{\alpha}_{i j}$.

Therefore, from (5), (6a) and (6b) we can respectively write

$$
\begin{aligned}
& \lambda_{i j}\left\|\boldsymbol{x}_{i}-\boldsymbol{s}_{j}\right\|=\eta d_{0}, \\
& \boldsymbol{c}_{i j}^{T}\left(\boldsymbol{x}_{i}-\boldsymbol{s}_{j}\right)=0, \\
& \boldsymbol{k}^{T}\left(\boldsymbol{x}_{i}-\boldsymbol{s}_{j}\right)=\left\|\boldsymbol{x}_{i}-\boldsymbol{s}_{j}\right\| \cos \left(\alpha_{i j}\right),
\end{aligned}
$$

where $\lambda_{i j}=10^{-\frac{L_{i j}}{10 \gamma}}, \eta=10^{-\frac{L_{0}}{10 \gamma}}, \boldsymbol{c}_{i j}=\left[-\sin \left(\phi_{i j}\right), \cos \left(\phi_{i j}\right)\right.$, $0]^{T}$ and $\boldsymbol{k}=[0,0,1]^{T}$. Applying the least squares approach to $(5),(6 a)$ and $(6 b)$, result in

$$
\begin{aligned}
\hat{\boldsymbol{X}}= & \underset{\boldsymbol{X}}{\arg \min } \sum_{(i, j) \in \mathcal{E}}\left(\lambda_{i j}\left\|\boldsymbol{x}_{i}-\boldsymbol{s}_{j}\right\|-\eta d_{0}\right)^{2} \\
& +\sum_{(i, j) \in \mathcal{E}}\left(\boldsymbol{c}_{i j}^{T}\left(\boldsymbol{x}_{i}-\boldsymbol{s}_{j}\right)\right)^{2} \\
& +\sum_{(i, j) \in \mathcal{E}}\left(\boldsymbol{k}^{T}\left(\boldsymbol{x}_{i}-\boldsymbol{s}_{j}\right)-\left\|\boldsymbol{x}_{i}-\boldsymbol{s}_{j}\right\| \cos \left(\alpha_{i j}\right)\right)^{2} .
\end{aligned}
$$


Notice that (8) is not convex due to the norm terms incorporating targets locations. Hence, instead of tackling (8) directly in the Cartesian space, we apply a simple conversion to the spherical, a more natural space due to acquired AOA observations [22]. To do so, we express $\boldsymbol{x}_{i}-\boldsymbol{s}_{j}=r_{i j} \boldsymbol{u}_{i j}$, with $r_{i j} \geq 0$ and $\left\|\boldsymbol{u}_{i j}\right\|=1$, which allows us to express $\left\|\boldsymbol{x}_{i}-\boldsymbol{s}_{j}\right\|=r_{i j}$. In order to define the unit vector, we simply take advantage of the available AOA observations, i.e., we have that $\boldsymbol{u}_{i j}=\left[\cos \left(\phi_{i j}\right) \sin \left(\alpha_{i j}\right), \sin \left(\phi_{i j}\right) \sin \left(\alpha_{i j}\right), \cos \left(\alpha_{i j}\right)\right]^{T}$ is the estimated unit direction vector. To revert the process and go back to the Cartesian space, it suffices to multiply the two equations by $\boldsymbol{u}_{i j}^{T} \boldsymbol{u}_{i j}=1$. Hence, by applying the described conversions to (7a) and (7c), we get

$$
\begin{aligned}
\lambda_{i j} \boldsymbol{u}_{i j}^{T} r_{i j} \boldsymbol{u}_{i j} & =\eta d_{0} \Leftrightarrow \lambda_{i j} \boldsymbol{u}_{i j}^{T}\left(\boldsymbol{x}_{i}-\boldsymbol{s}_{j}\right)=\eta d_{0}, \\
\boldsymbol{k}^{T} r_{i j} \boldsymbol{u}_{i j} & =\boldsymbol{u}_{i j}^{T} r_{i j} \boldsymbol{u}_{i j} \cos \left(\alpha_{i j}\right) \\
& \Leftrightarrow\left(\cos \left(\alpha_{i j}\right) \boldsymbol{u}_{i j}-\boldsymbol{k}\right)^{T}\left(\boldsymbol{x}_{i}-\boldsymbol{s}_{j}\right)=0 .
\end{aligned}
$$

Note that both RSS and AOA nearby links are trusted more than the remote ones [18]. Thus, in order to assign more confidence to nearby links, we introduce weights, $\boldsymbol{w}=\left[\sqrt{w_{i j}}\right]^{T}$, with $w_{i j}=1-\widehat{d}_{i j} / \sum_{(i, j) \in \mathcal{E}} \widehat{d}_{i j}$. Then, by following the WLS criterion, an estimate of $\boldsymbol{X}$ can be obtained by solving

$$
\begin{aligned}
\hat{\boldsymbol{X}}= & \underset{\boldsymbol{X}}{\arg \min } \sum_{(i, j) \in \mathcal{E}} w_{i j}\left(\lambda_{i j} \boldsymbol{u}_{i j}^{T}\left(\boldsymbol{x}_{i}-\boldsymbol{s}_{j}\right)-\eta d_{0}\right)^{2} \\
& +\sum_{(i, j) \in \mathcal{E}} w_{i j}\left(\boldsymbol{c}_{i j}^{T}\left(\boldsymbol{x}_{i}-\boldsymbol{s}_{j}\right)\right)^{2} \\
& +\sum_{(i, j) \in \mathcal{E}} w_{i j}\left(\left(\cos \left(\alpha_{i j}\right) \boldsymbol{u}_{i j}-\boldsymbol{k}\right)^{T}\left(\boldsymbol{x}_{i}-\boldsymbol{s}_{j}\right)\right)^{2} .
\end{aligned}
$$

Notice that all three sums are quadratic with respect to $\boldsymbol{X}$. Hence, (10) can be rewritten in an equivalent vector form as

$$
\underset{\boldsymbol{x}}{\operatorname{minimize}}\|\boldsymbol{W}(\boldsymbol{A} \boldsymbol{x}-\boldsymbol{b})\|^{2}
$$

where $\boldsymbol{x}=\left[\boldsymbol{x}_{1}^{T}, \ldots, \boldsymbol{x}_{M}^{T}\right]^{T}, \quad\left(\boldsymbol{x} \in \mathbb{R}^{3 M \times 1}\right), \quad \boldsymbol{W}=\boldsymbol{I}_{3} \otimes \operatorname{diag}$ $(\boldsymbol{w})$, with $\otimes$ denoting the Kronecker product, and

$$
\begin{aligned}
& \text { - } \boldsymbol{A}_{t, 3(i-1)+1: 3 i}=\lambda_{i j} \boldsymbol{u}_{i j}^{T}, \boldsymbol{b}_{t}=\lambda_{i j} \boldsymbol{u}_{i j}^{T} \boldsymbol{a}_{j}+\eta d_{0}, \\
& \forall(i, j) \in \mathcal{E}_{A}, t=1, \ldots,\left|\mathcal{E}_{A}\right| ; \\
& \text { - } \boldsymbol{A}_{t, 3(i-1)+1: 3 i}=\boldsymbol{c}_{i j}^{T}, \boldsymbol{b}_{t}=\boldsymbol{c}_{i j}^{T} \boldsymbol{a}_{j}, \\
& \forall(i, j) \in \mathcal{E}_{A}, t=\left|\mathcal{E}_{A}\right|+1, \ldots, 2\left|\mathcal{E}_{A}\right| ; \\
& \text { - } \boldsymbol{A}_{t, 3(i-1)+1: 3 i}=\left(\cos \left(\alpha_{i j}\right) \boldsymbol{u}_{i j}-\boldsymbol{k}\right)^{T}, \\
& \boldsymbol{b}_{t}=\left(\cos \left(\alpha_{i j}\right) \boldsymbol{u}_{i j}-\boldsymbol{k}\right)^{T} \boldsymbol{a}_{j}, \\
& \forall(i, j) \in \mathcal{E}_{A}, t=2\left|\mathcal{E}_{A}\right|+1, \ldots, 3\left|\mathcal{E}_{A}\right| ; \\
& \text { - } \boldsymbol{A}_{t,[3(i-1)+1: 3 i, 3(j-1)+1: 3 j]}=\left[\lambda_{i j} \boldsymbol{u}_{i j}^{T},-\lambda_{i j} \boldsymbol{u}_{i j}^{T}\right], \boldsymbol{b}_{t}=\eta d_{0}, \\
& \forall(i, j) \in \mathcal{E}_{T}, t=3\left|\mathcal{E}_{A}\right|+1, \ldots, 3\left|\mathcal{E}_{A}\right|+\left|\mathcal{E}_{T}\right| ; \\
& \text { - } \boldsymbol{A}_{t,[3(i-1)+1: 3 i, 3(j-1)+1: 3 j]}=\left[\boldsymbol{c}_{i j}^{T},-\boldsymbol{c}_{i j}^{T}\right], \boldsymbol{b}_{t}=0, \\
& \forall(i, j) \in \mathcal{E}_{T}, t=3\left|\mathcal{E}_{A}\right|+\left|\mathcal{E}_{T}\right|+1, \ldots, 3\left|\mathcal{E}_{A}\right|+2\left|\mathcal{E}_{T}\right| ; \\
& \text { - } \boldsymbol{A}_{t,[3(i-1)+1: 3 i, 3(j-1)+1: 3 j]}=\left[\left(\cos \left(\alpha_{i j}\right) \boldsymbol{u}_{i j}-\boldsymbol{k}\right)^{T},\right. \\
& \left.-\left(\cos \left(\alpha_{i j}\right) \boldsymbol{u}_{i j}-\boldsymbol{k}\right)^{T}\right], \boldsymbol{b}_{t}=0, \\
& \forall(i, j) \in \mathcal{E}_{T}, t=3\left|\mathcal{E}_{A}\right|+2\left|\mathcal{E}_{T}\right|+1, \ldots, 3\left|\mathcal{E}_{A}\right|+3\left|\mathcal{E}_{T}\right|,
\end{aligned}
$$

with $\mathcal{E}_{A}$ and $\mathcal{E}_{T}$ denoting the tuple sets of all target/anchor and target/target edges, and $|\bullet|$ being the cardinality of a set.
TABLE I

Average EXeCUtion Time of the CONSIDERED Algorithms

\begin{tabular}{|c||c|c|c|}
\hline Algorithm & WLS & LLS in [19] & SDP in [18] \\
\hline Time (s) & 0.0362 & 0.0386 & 15.9 \\
\hline
\end{tabular}

The solution to (11) is given in closed-form as

$$
\hat{\boldsymbol{x}}=\left(\boldsymbol{A}^{T} \boldsymbol{W}^{T} \boldsymbol{W} \boldsymbol{A}\right)^{-1}\left(\boldsymbol{A}^{T} \boldsymbol{W}^{T} \boldsymbol{b}\right) .
$$

In the remaining text, (11) is referred to as "WLS".

\section{COMPLEXITY ANALYSIS}

To analyze the worst case computational complexity, a fully connected network is studied and only the dominating terms are considered, expressed as a function of $N$ and $M$. Complexity of the proposed WLS and LLS in [19] is linear in the number of edges, i.e., it is of order $\mathcal{O}\left(M N+\frac{M(M-1)}{2}\right)$. This is substantially lower than the complexity of the SDP algorithm in [18] which is of order $\mathcal{O}\left(M^{4.5}\left(N+\frac{M}{2}\right)^{2}\right)$. To better illustrate this result, the reader is referred to Table I, where the average execution time of the algorithms is given.

The considered setting was $N=6, M=30, R=5 \mathrm{~m}$, and the remaining parameters were set as described in Section V. The results in Table I show that in fact there is a tremendous difference in terms of the execution time between the two linear algorithms and the SDP one, as expected.

\section{NUMERICAL RESULTS}

This section validates the performance of the new algorithm through computer simulations. A connected network is considered, where the observations were generated through (1) and (2) with relatively high noise powers. All sensors were deployed randomly inside a box with an edge length $B=10 \mathrm{~m}$ in each Monte Carlo $\left(M_{c}\right)$ run. The reference distance is set to $d_{0}=1 \mathrm{~m}$, the reference path loss to $L_{0}=40 \mathrm{~dB}$, and the PLE was fixed to $\gamma=2.5$. However, to account for a realistic measurement model mismatch and test the robustness of the new algorithms to imperfect knowledge of the PLE, the true PLE was drawn from a uniform distribution on the interval $[2.2,2.8]$, i.e., $\gamma_{i j} \sim \mathcal{U}[2.2,2.8], \forall(i, j) \in \mathcal{E}$. Furthermore, $\sigma_{n_{i j}}=10 \mathrm{~dB}$ and $\kappa_{i j}=25.9$, which corresponds to the circular standard deviation of $\sigma_{m_{i j}}=\sigma_{v_{i j}}=\sigma_{i j}=8 \mathrm{deg}$, since $\sigma_{i j}^{2}=1-I_{1}\left(\kappa_{i j}\right) / I_{0}\left(\kappa_{i j}\right)$ [21]. The main performance metric is the normalized root mean square error (NRMSE), NRMSE $=\sqrt{\sum_{j=1}^{M_{c}} \sum_{i=1}^{M} \frac{\left\|\boldsymbol{x}_{i j}-\widehat{\boldsymbol{x}}_{i j}\right\|^{2}}{M M_{c}}}$, where $\widehat{\boldsymbol{x}}_{i j}$ is the estimate of the true target location, $\boldsymbol{x}_{i j}$, in the $j$-th $M_{c}$ run.

The performance of the WLS estimator is compared to the existing SDP in [18] and LLS in [19] for RSS-AOA localization in cooperative WSNs. Furthermore, the results obtained by its counterpart that takes advantage of AOA-only observations, denoted by "LS $\mathrm{AOA}_{\mathrm{AO}}$ ", are included also in order to show the benefit of a hybrid approach in comparison to the classical one. ${ }^{2}$ Finally, the Cramer-Rao lower bound (CRLB) [18] is also included in all figures.

\footnotetext{
${ }^{1}$ Note that the proposed estimator can be adapted to the case where $P_{T}$, i.e., $L_{0}$ is not known; it suffices to consider $\eta$ in (10) as an estimation variable and add an extra column in $\boldsymbol{A}$ with regards to the coefficients alongside it.

${ }^{2}$ Note that the other counterpart, RSS-only, can not be implemented in the same manner, since the proposed approach requires directional information.
} 


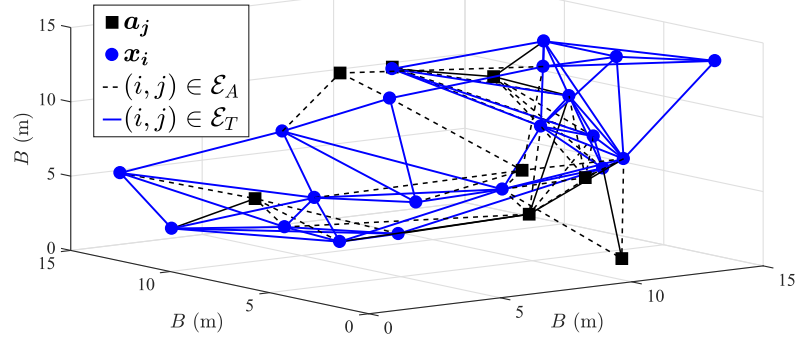

(a) Network example

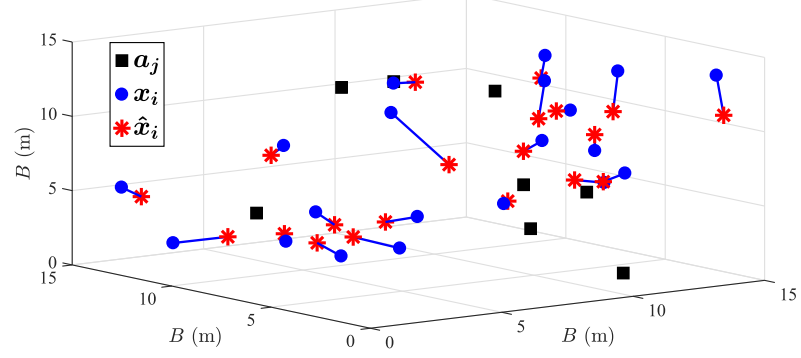

(b) Estimation via WLS

Fig. 2. Illustration of the estimation process by using the proposed WLS algorithm for $N=8, M=20$ and $R=8 \mathrm{~m}$.

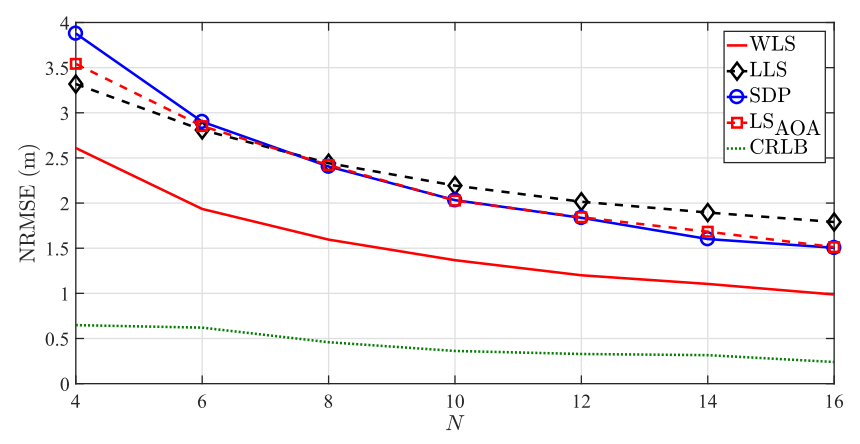

Fig. 3. NRMSE versus $N: M=30, R=4 \mathrm{~m}, M_{c}=50000$.

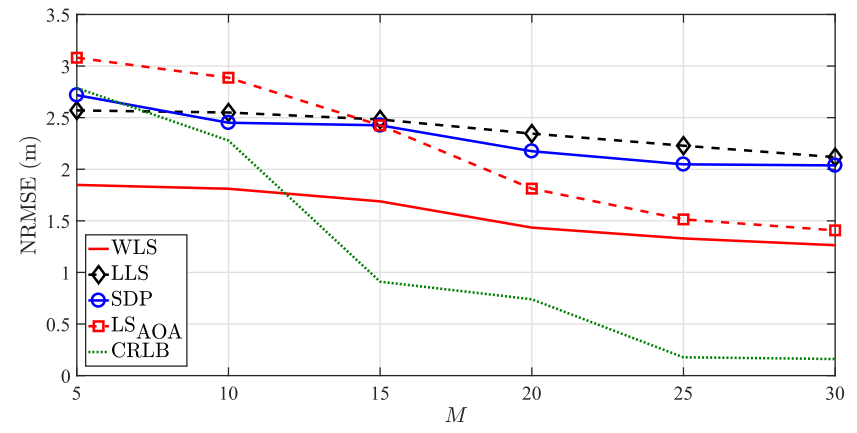

Fig. 4. NRMSE versus $M: N=7, R=5 \mathrm{~m}, M_{c}=50000$.

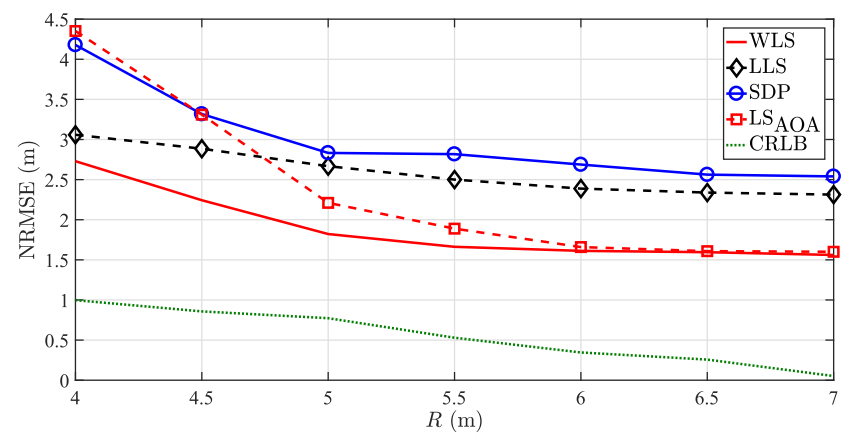

Fig. 5. NRMSE versus $R(\mathrm{~m}): N=5, M=20, M_{c}=50000$.

Fig. 2 illustrates one particular output by WLS. It can be seen from the figure that the accuracy is very good in general, and that even the targets with no anchor neighbors (e.g., the two far-right top ones) can be localized fairly well.

Figs. 3, 4 and 5 illustrate the NRMSE (m) versus $N, M$ and $R(\mathrm{~m})$ comparison, respectively. Interestingly, Fig. 3 shows

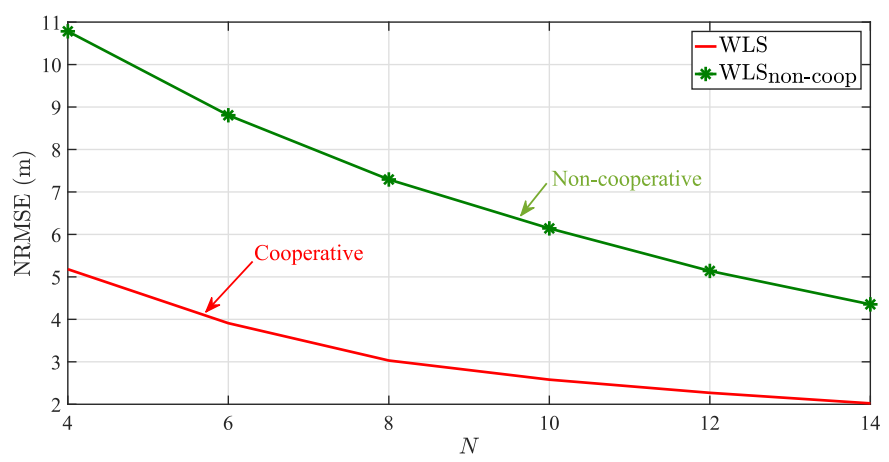

Fig. 6. NRMSE versus $N: M=10, R=8 \mathrm{~m}, \sigma_{n_{i}}=6 \mathrm{~dB}, \kappa_{i j}=16.67$, $B=15 \mathrm{~m}, M_{c}=50000$.

that the performance margin between WLS and LLS does not decrease as $N$ increases. This is somewhat surprising, since when $N$ is increased the set $\mathcal{E}_{T}$ grows in general, allowing good performance for any algorithm. This means that LLS does not exploit the additional information as well as WLS and SDP do. Figs. 4 and 5 confirm the utility of the cooperative concept, rendering higher accuracy as $M$ and $R(\mathrm{~m})$ are increased in general. All three figures show that WLS offers a significant gain over LLS and SDP, especially for low quantities of the respective parameters.

Finally, to validate the cooperative concept, the comparison of the proposed WLS with itself, when no node cooperation is allowed and each target has anchor neighbors exclusively, is presented in Fig. 6. From it, it is clear that the cooperative concept brings advantage over the non-cooperative one, especially for low $N$, as expected. This result confirms that the extra information from target/target connections gathered within the network is handled properly.

\section{CONCLUSIONS}

In this letter, a novel RSS-AOA-based algorithm for simultaneous localization of multiple targets was presented. Unlike some existing algorithms that resort to sophisticated mathematical tools or do not always provide a feasible solution, a more natural approach was taken here. By exploiting the available AOA measurements, a switch from Cartesian to spherical coordinates was performed, and the originally non-convex measurement models were approximated by linear ones. This allowed us to efficiently exploit the geometry of the problem and solve it in a closed-form. Our solution is not only linear in terms of computational complexity, it also provides higher localization accuracy than the state-of-the-art methods. 


\section{REFERENCES}

[1] S. Tomic, M. Beko, and R. Dinis, "RSS-based localization in wireless sensor networks using convex relaxation: Noncooperative and cooperative schemes," IEEE Trans. Veh. Technol., vol. 64, no. 5, pp. 2037-2050, May 2015.

[2] M. Z. Win et al., "Network localization and navigation via cooperation," IEEE Commun. Mag., vol. 49, no. 5, pp. 56-62, May 2011.

[3] M. Laaraiedh, L. Yu, S. Avrillon, and B. Uguen, "Comparison of hybrid localization schemes using RSSI, TOA, and TDOA," in Proc. 17th Eur. Wireless Sustain. Wireless Technologies, Vienna, Austria, 2011, pp. 1-5.

[4] Y. Wang, F. Zheng, M. Wiemeler, W. Xiong, and T. Kaiser, "Reference selection for hybrid TOA/RSS linear least squares localization," in Proc. IEEE 78th Veh. Technol. Conf. Fall, Las Vegas, NV, USA, Sep. 2013, pp. $1-5$.

[5] A. Coluccia and A. Fascista, "On the hybrid TOA/RSS range estimation in wireless sensor networks," IEEE Trans. Wireless Commun., vol. 17, no. 1, pp. 361-371, Jan. 2018.

[6] L. Cong and W. Zhuang, "Hybrid TDOA/AOA mobile user location for wide-band CDMA cellular systems," IEEE Trans. Wireless Commun., vol. 1, no. 3, pp. 439-447, Jul. 2002.

[7] V. Y. Zhang, A. K. Wong, K. T. Woo, and R. W. Ouyang, "Hybrid TOA/AOA-based mobile localization with and without tracking in CDMA cellular networks," in Proc. IEEE Wireless Commun. Netw. Conf., Sydney, NSW, USA, Apr. 2010, pp. 1-6.

[8] S. Tomic and M. Beko, "A bisection-based approach for exact target localization in NLOS environments," Signal Process., vol. 143, pp. 328335, Feb. 2018.

[9] S. Safavi, U. A. Khan, S. Kar, and J. M. F. Moura, "Distributed localization: A linear theory," Proc. IEEE, vol. 106, no. 7, pp. 1204-1223, Jul. 2018.

[10] K. Yu, "3-D localization error analysis in wireless networks," IEEE Trans. Wireless Commun., vol. 6, no. 10, pp. 3473-3481, Oct. 2007.

[11] S. Wang, B. R. Jackson, and R. Inkol, "Hybrid RSS/AOA emitter location estimation based on least squares and maximum likelihood criteria," in Proc. 26th Biennial Symp. Commun., Jun. 2012, pp. 24-29.
[12] S. Tomic, M. Beko, R. Dinis, and P. Montezuma, "A closed-form solution for RSS/AoA target localization by spherical coordinates conversion," IEEE Wireless Commun. Lett., vol. 5, no. 6, pp. 680-683, Dec. 2016.

[13] L. Gazzah, L. Najjar, and H. Besbes, "Hybrid RSS/AOA hypothesis test for NLOS/LOS Base station discrimination and location error mitigation," Trans. Emerg. Telecommun. Technol., vol. 27, no. 5, pp. 629-639, May 2016.

[14] M. W. Khan, N. Salman, A. H. Kemp, and L. Mihaylova, "Localisation of sensor nodes with hybrid measurements in wireless sensor networks," Sensors, vol. 16, no. 7, pp. 1-16, Jul. 2016.

[15] S. Tomic, M. Beko, and R. Dinis, "Distributed RSS-AoA based localization with unknown transmit powers," IEEE Wireless Commun. Lett., vol. 5, no. 4, pp. 392-395, Aug. 2016.

[16] S. Tomic, M. Beko, R. Dinis, and P. Montezuma, "Distributed algorithm for target localization in wireless sensor networks using RSS and AoA measurements," Pervasive Mobile Comput., vol. 37, pp. 63-77, Oct. 2016.

[17] P. Biswas, H. Aghajan, and Y. Ye, "Semidefinite programming algorithms for sensor network localization using angle of arrival information," in Proc. 39th Asilomar Conf. Signals, Syst. Comput., Oct. 2005 , pp. $220-224$

[18] S. Tomic, M. Beko, and R. Dinis, "3-D target localization in wireless sensor network using RSS and AoA measurement," IEEE Trans. Veh. Technol., vol. 66, no. 4, pp. 3197-3210, Apr. 2017.

[19] M. W. Khan, N. Salman, and A. H. Kemp, "Optimised hybrid localisation with cooperation in wireless sensor networks," IET Signal Process. vol. 11, no. 3, pp. 341-348, May 2017.

[20] K. V. Mardia Statistics of Directional Data. London, England: Academic, 1972.

[21] C. Forbes, M. E. Evans, N. Hastings, and B. Peacock, Statistical Distributions, 4th ed. Hoboken, NJ, USA: Wiley, 2011.

[22] J. Yin, Q. Wan, S. Yang, and K. C. Ho, "A simple and accurate TDOAAOA localization method using two stations," IEEE Signal Process. Lett., vol. 23, no. 1, pp. 144-148, Jan. 2016. 